\title{
Co-Occurrence of Myeloid Sarcoma of the Lymph Node and Acute Monocytic Myeloid Leukemia: A Case Report and Literature Review
}

\author{
Misbahuddin Khaja ${ }^{a} \quad$ Toolsie Omesh $^{\mathrm{a}}$ Masooma Niazi ${ }^{\mathrm{b}} \quad$ Danial Shaikh $^{\mathrm{c}}$ \\ Sitarama Arvind Mudunuru ${ }^{c}$ Olga Alexandra Reyes ${ }^{c}$ Sandeep Malik ${ }^{d}$ \\ aDivision of Pulmonary and Critical Care Medicine, Bronx Care Health System, Affiliated \\ with Icahn School of Medicine at Mount Sinai, Bronx, NY, USA; 'bivision of Pathology, \\ Bronx Lebanon Hospital Center, Affiliated with Icahn School of Medicine at Mount Sinai, \\ Bronx, NY, USA; 'Department of Medicine, Bronx Care Health System, Affiliated with Icahn \\ School of Medicine at Mount Sinai, Bronx, NY, USA; division of Hematology and \\ Oncology, Bronx Care Health System, Affiliated with Icahn School of Medicine at Mount \\ Sinai, Bronx, NY, USA
}

\section{Keywords}

Myeloid Sarcoma $\cdot$ Acute Monocytic Myeloid Leukemia $\cdot$ Myeloid cell tumor · Myeloperoxidase reaction · Flow cytometry

\begin{abstract}
Background: Acute myeloid leukemia (AML) is the most common leukemia in adults. According to the French-American-British (FAB) system, monocytic leukemia is classified as M5. Myeloid sarcoma further occurs in $3-5 \%$ of AML. This is defined as an extramedullary tumor of myeloid cells in the lymph nodes, soft tissues, periosteum, bone, central nervous system (CNS), spinal cord, intestine, mediastinum, prostate, uterus, or ovaries. Case Presentation: Here, we describe the case of a 29-year-old female who presented with fever, swelling of gums, neck
\end{abstract}


pain, and weakness, which had persisted for 1 week. The patient had a white blood cell (WBC) count of $53.5 \mathrm{~K} / \mu \mathrm{L}$, and a peripheral smear revealed a myeloid blast cell (blast) percentage of $8 \%$. Computed tomography (CT) of the neck indicated lymphadenopathy. Fine needle aspiration of the cervical lymph node showed groups of atypical immature myeloid cells, mixed with occasional megakaryocytes, and infiltration of eosinophilic myeloid cells into the lymph node, consistent with myeloid sarcoma. Flow cytometry analysis revealed intermediate to large circulating blasts, with irregular nuclei, fine chromatin, and distinct nucleoli, indicative of AML, with monocytic differentiation. The patient responded well to chemotherapy with fludarabine, cytarabine, granulocyte colony stimulating factor (G-CSF), and idarubicin; WBC counts returned to normal and patient was discharged to home. Conclusion: Myeloid sarcoma of the lymph node is a rare co-occurrence with AML. Results of our study are consistent with the conclusion that early diagnosis and appropriate treatment improve survival.

\section{Introduction}

In adult patients, the most common leukemia is acute myeloid leukemia (AML). This disease is characterized by clonal expansion of myeloid blast cells (blasts), which do not differentiate into mature cells. The accumulation of these immature cells in tissues promotes increased production of malignant cells, leading to bleeding, anemia, and infection [1]. Myeloid sarcoma is tumor of immature myeloid cells occurring in extramedullary areas, such as the lymph nodes, soft tissues, periosteum, bone, central nervous system (CNS), spinal cord, intestine, mediastinum, prostate, uterus, or ovaries. These tumors may occur earlier than, or concurrent with, AML [2].

The World Health Organization (WHO) has classified myeloid neoplasm and acute leukemia into multiple different categories. These include myeloproliferative neoplasms, myeloid/lymphoid neoplasm with eosinophilia, myelodysplastic/myeloproliferative neoplasms, myelodysplastic syndromes (MDS), mastocytosis, myeloid neoplasm with germ line predisposition, acute myeloid leukemia, blastic plasmacytoid dendritic cell neoplasm, acute leukemias of ambiguous lineage, B-lymphoblastic leukemia/lymphoma, and T-lymphoblastic leukemia/lymphoma [3].

Incidence of Myeloid sarcoma is 3 to $5 \%$ in patient with AML [4]. Here we present a case of young female who has co-occurrence of myeloid sarcoma with AML.

\section{Case Presentation}

A 29-year-old female, with no past medical history, came in for evaluation of fever, swelling of gums, neck pain, and weakness that had persisted one week. She denied chills, rigor, dysphagia, odynophagia, abdominal pain, nausea or vomiting, and headache. No cough or shortness of breath was noted; although, she reported difficulty chewing her food due to pain in her gums. 
On physical examination, her vital signs were as follows: temperature of $100^{\circ} \mathrm{F}$, pulse rate of 90 beats per min, respiration rate of 12 breaths per minute, blood pressure of 110/70 $\mathrm{mmHg}$, and oxygen saturation of $98 \%$ on room air. Oral examination revealed generalized gingival swelling (Fig. 1A) and cervical lymphadenopathy; the lung, cardiovascular, and central nervous systems, as well as the abdomen, were within normal limits.

The patient's laboratory data on the day of admission showed a total white blood cell (WBC) count of $53.5 \mathrm{~K} / \mu \mathrm{L}$, a platelet count of $104 \mathrm{~K} / \mu \mathrm{L}$, hemoglobin of $10.8 \mathrm{~g} / \mathrm{dL}$, and hematocrit of $30.9 \%$ (Table 1). Her blood chemistry was within normal limits, except for lactate dehydrogenase, which was $408 \mathrm{U} / \mathrm{L}$. A peripheral smear further showed a blast cell percentage of $8 \%$. Chest radiograph revealed no acute findings, however, computed tomography (CT) of the neck showed extensive lymphadenopathy, with bilateral submandibular and internal jugular lymphadenopathy (Fig. 1B). Chest CT did not reveal lymphadenopathy, although CT of the abdomen showed splenomegaly.

Flow cytometry analysis of patient blood revealed circulating blasts, intermediate to large in size, with irregular nuclei, fine chromatin, distinct nucleoli, and a scant to moderate amount of cytoplasm, which accounted for approximately $40-50 \%$ of the total WBC count. Scattered myelocytes and nucleated red blood cells are also noted. On further evaluation, these analyses revealed a dim CD45 blast-gated population, expressing dim CD117, CD4, CD11c, CD13, CD33, CD30, myeloperoxidase (MPO), and HLA-DR, which accounted for approximately $42 \%$ of total gated events (Fig. 2). Fluorescence in situ hybridization (FISH) for promyelocytic leuke$\mathrm{mia} /$ retinoic acid receptor alpha (PML/RARA) was negative, and the final diagnosis was acute myeloid leukemia, with monocytic differentiation.

Fine needle aspiration of the cervical lymph node was performed, which showed groups of atypical, immature myeloid cells, mixed with occasional megakaryocytes, and eosinophilic myeloid cells infiltrating the lymph node, consistent with myeloid sarcoma (extramedullary myeloid neoplasm/chloroma) (Fig. 3A-C). A bone marrow aspirate smear with WrightGiemsa stain subsequently showed hypercellular marrow, with $89 \%$ blasts/promonocytes, $1 \%$ segmented neutrophils, $2 \%$ monocytes, $2 \%$ erythroid precursors, $3 \%$ lymphocytes, $3 \%$ plasma cells, and $0 \%$ promyelocytes, myelocytes, metamyelocytes, bands, and basophils/eosinophils and precursors. Bone marrow biopsy was performed at the right posterior iliac crest, and trephine biopsy showed marked hypercellular marrow, containing $>95 \%$ immature myeloid/monocytic cells. The bony trabeculae were unremarkable, with an absence of granuloma, which is consistent with AML with monocytic features (Fig. 3D).

The patient was seen by hematology services and on day 4 of presentation she was started on chemotherapy of fludarabine, cytarabine, granulocyte colony stimulating factor (G-CSF), and idarubicin. During this treatment course, her WBC count improved (Table 1). She completed this course of chemotherapy and was discharged to home, with follow-ups at the hematology clinic.

\section{Discussion}

AML, also known as acute granulocytic leukemia, acute myeloblastic leukemia, and acute nonlymphocytic leukemia, is a type of malignancy that originates from cells in the bone marrow. The WHO has classified AML in to six main groups, including AML with recurrent genetic 
abnormalities, AML with myelodysplasia-related features, therapy-related AML, myeloid sarcoma, myeloid proliferation related to Down Syndrome, and AML not otherwise specified [5]. The French-American-British (FAB) classification system has further classified AML in to subtypes based on cytochemical staining, immunophenotype, and morphology. These types can be determined by flow cytometry or immunohistochemistry and include granulocytic leukemias, M0, M1, M2, and M3, and type M4, which has both granulocytic and monocytic cells, at least 20\% of which are monocytic. M5 is monocytic, M6 involves megaloblastic changes, and M7 is acute megakaryocytic leukemia [2].

AML occurs in older populations but can also be seen in woman of child-bearing age. Known risk factors for development of AML include increasing age, a previous history of cancer treatment, exposure to radiation, chemicals, cigarette smoking, genetic disorders, and history of other blood disorders, such as polycythemia vera, myelofibrosis, myelodysplasia, thrombocytopenia; it also more common in men then in women [6].

AML can present as bone pain, lethargy, fatigue, pallor, shortness of breath, bruising, nosebleeds, and bleeding from the gums. When skin lesions are present, these can be nodular and violaceous in color. Leukemic infiltration of the skin can occur in monocytic or myelomonocytic AML. Headache, visual changes, and cranial nerve involvement may also be seen in acute monocytic leukemia, and gingival hypertrophy, arising due to leukemic involvement, is commonly observed in the monocytic subtype of AML [7].

Myeloid sarcoma co-occurs in 3-5\% of patients with AML. In 1811, Burns first described myeloid sarcoma as an extramedullary tumor of myeloid cells, which are immature. In 1853, it was characterized as a chloroma because of the green color associated with MPO, and later, it was known as granulocytic sarcoma [8]. Myeloid sarcoma is common in pediatric patients, with an incidence of $30 \%$ in children with AML and no sex predominance. Extramedullary sites of involvement for Myeloid sarcoma include the lymph nodes, soft tissues, periosteum, bone, and rare sites such as the CNS, spinal cord, intestine, mediastinum, prostate, uterus, and ovaries $[9,10]$.

Myeloid sarcoma can present in three patterns in patients with AML. In some cases, it occurs during the active phase of leukemia, whereas in others, it manifests as relapse after remission. MS may also present before AML is diagnosed [11]. Myeloid sarcoma should be suspected if eosinophilic myelocytes are observed using hematoxylin and eosin staining. Diagnosis is established by identifying myeloid cells, using MPO stain, immunophenotyping of tissue, or flow cytometry. MPO, CD68, and lysozyme are the most common immunohistochemical markers of myeloid sarcoma [12]. In the FAB M4/M5 type of AML, those with certain cytogenetic abnormalities, including a balanced translocation between chromosomes 18 and 21 ( $\mathrm{t}(8 ; 21))$ and inversion $16(\mathrm{inv}(16)), \mathrm{CD}^{2} 6^{+}$blasts, $11 \mathrm{q}$ abnormalities, a recent history of allogenic stem cell transplant, and cellular immune dysfunction have higher incidence of Myeloid sarcoma [13].

Prognosis does not change with occurrence of Myeloid sarcoma in AML patients. Extramedullary blast crises of chronic myeloid leukemia (CML) or acute leukemic transformations of myeloproliferative disorders should be considered as differential diagnosis for myeloid sarcoma. A peripheral smear should also be examined for circulating leukemic blasts, followed by definitive bone marrow aspiration and biopsy. Peripheral smears may have circulating myeloblasts, which have a high nuclear to cytoplasmic ratio and nucleoli, and Auer rods can be seen as pink or red rod like granular structures in the cytoplasm. The MPO 
reaction can further be performed to show that blasts are myeloid. Flow cytometry is required to identify myeloblasts by patterns of surface antigen expression, with the majority expressing CD34, HLA-DR, CD117, CD13, and CD33. Morphologic evaluation, immunophenotype, karyotype analysis, and molecular genetic testing are needed for definitive diagnosis of acute leukemia [14].

Recommend treatment for Myeloid sarcoma is similar to treatment of AML, and patients with Myeloid sarcoma, if left untreated, will eventually develop AML. The Myeloid sarcoma population has good overall survival on standard AML chemotherapy. In a study by Antic et al., clinicians used anthracycline and cytarabine as chemotherapeutic agents, followed by auto or allogenic stem cell transplantation. They found that patients who received bone marrow transplants had longer survival than those who received conventional therapy [15]. Radiation therapy and surgical resection are also considered for local tumor. In this case, our patient responded to a chemotherapy regimen of fludarabine, cytarabine, G-CSF, and idarubicin, with subsequent normalization of white cell count.

\section{Conclusion}

Myeloid sarcoma of the lymph node is rare presentation of AML. Here, we describe the case of a patient with a co-occurrence of myeloid sarcoma with acute monocytic myeloid leukemia. The patient responded well to chemotherapy, consistent with the observation that early diagnosis and appropriate treatment will improve survival.

\section{Acknowledgements}

No funding was provided for the production of this case report.

\section{Statement of Ethics}

The authors have no ethical conflicts to disclose.

\section{Disclosure Statement}

The authors have no conflicts of interest to declare.

\section{Funding Sources}

No financial support was used for this case report. 


\section{Author Contributions}

M Khaja, S Malik and T Omesh searched the literature and wrote the manuscript. M Khaja conceived and edited the manuscript. M Khaja supervised the patient treatment, critically revised and edited the manuscript. 0 Reyes, S Arvind and D Shaikh were also involved in patient care and medical management. M Niazi gave us input about pathology. All authors have made significant contributions to the manuscript and have reviewed it before submission. All authors have confirmed that the manuscript is not under consideration for review at any other Journal. All authors have read and approved the final manuscript.

\section{References}

1 Vardiman JW, Thiele J, Arber DA, Brunning RD, Borowitz MJ, Porwit A, et al. The 2008 revision of the World Health Organization (WHO) classification of myeloid neoplasms and acute leukemia: rationale and important changes. Blood. 2009 Jul;114(5):937-51.

2 Catovsky D, Matutes E, Buccheri V, Shetty V, Hanslip J, Yoshida N, et al. A classification of acute leukaemia for the 1990s. Ann Hematol. 1991 Feb;62(1):16-21.

3 Arber DA, Orazi A, Hasserjian R, Thiele J, Borowitz MJ, Le Beau MM, et al. The 2016 revision to the World Health Organization classification of myeloid neoplasms and acute leukemia. Blood. 2016 May;127(20):2391-405.

4 Yilmaz AF, Saydam G, Sahin F, Baran Y. Granulocytic sarcoma: a systematic review. Am J Blood Res. 2013 Dec;3(4):265-70.

5 Grimwade D, Hills RK, Moorman AV, Walker H, Chatters S, Goldstone AH, et al.; National Cancer Research Institute Adult Leukaemia Working Group. Refinement of cytogenetic classification in acute myeloid leukemia: determination of prognostic significance of rare recurring chromosomal abnormalities among 5876 younger adult patients treated in the United Kingdom Medical Research Council trials. Blood. 2010 Jul;116(3):354-65.

6 Deschler B, Lübbert M. Acute myeloid leukemia: epidemiology and etiology. Cancer. 2006 Nov;107(9):2099107.

7 Meyers CA, Albitar M, Estey E. Cognitive impairment, fatigue, and cytokine levels in patients with acute myelogenous leukemia or myelodysplastic syndrome. Cancer. 2005 Aug;104(4):788-93.

8 Burns A. Observations of surgical anatomy, head and neck. Thomas Royce and co, Edinburgh 1811; 364-366.

9 Klco JM, Welch JS, Nguyen TT, Hurley MY, Kreisel FH, Hassan A, et al. State of the art in myeloid sarcoma. Int J Lab Hematol. 2011 Dec;33(6):555-65.

10 Yamauchi K, Yasuda M. Comparison in treatments of nonleukemic granulocytic sarcoma: report of two cases and a review of 72 cases in the literature. Cancer. 2002 Mar;94(6):1739-46.

11 Neiman RS, Barcos M, Berard C, Bonner H, Mann R, Rydell RE, et al. Granulocytic sarcoma: a clinicopathologic study of 61 biopsied cases. Cancer. 1981 Sep;48(6):1426-37.

12 Bakst RL, Tallman MS, Douer D, Yahalom J. How I treat extramedullary acute myeloid leukemia. Blood. 2011 Oct;118(14):3785-93.

13 Paydas S, Zorludemir S, Ergin M. Granulocytic sarcoma: 32 cases and review of the literature. Leuk Lymphoma. 2006 Dec;47(12):2527-41.

14 Arber DA, Borowitz MJ, Cessna M, Etzell J, Foucar K, Hasserjian RP, et al. Initial Diagnostic Workup of Acute Leukemia: Guideline From the College of American Pathologists and the American Society of Hematology. Arch Pathol Lab Med. 2017 Oct;141(10):1342-93.

15 Antic D, Elezovic I, Milic N, Suvajdzic N, Vidovic A, Perunicic M, et al. Is there a "gold" standard treatment for patients with isolated myeloid sarcoma? Biomed Pharmacother. $2013 \mathrm{Feb}$;67(1):72-7. 


\section{Case Reports in Oncology}

Khaja et al.: Co-Occurrence of Myeloid Sarcoma of the Lymph Node and Acute

Monocytic Myeloid Leukemia: A Case Report and Literature Review

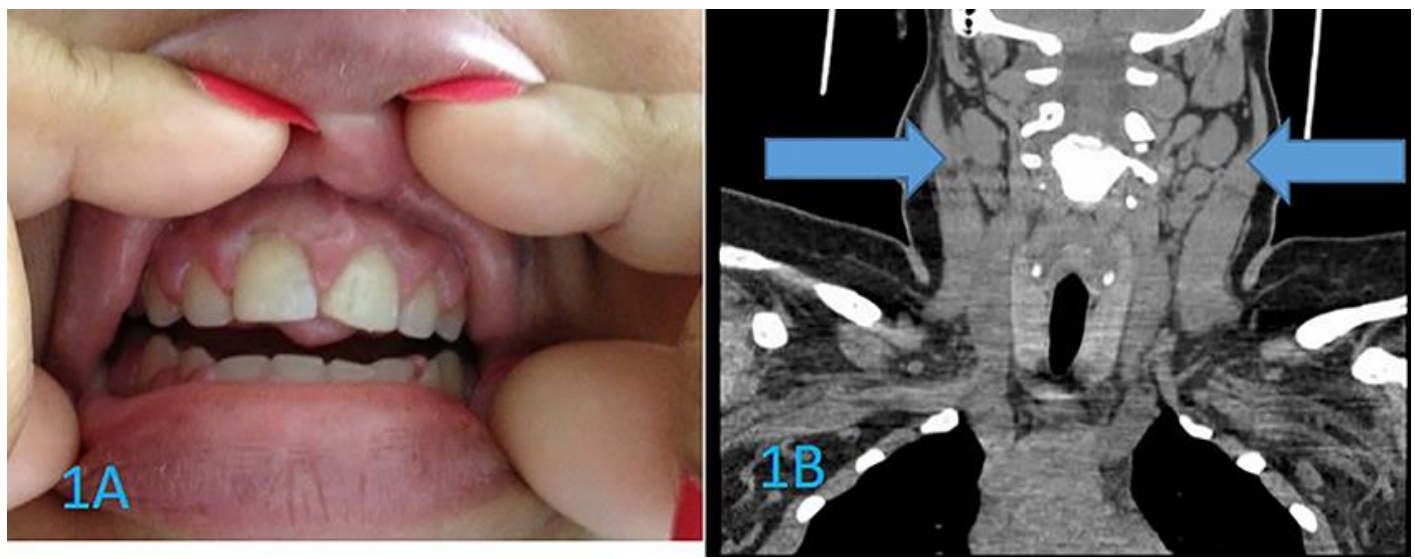

Fig. 1. A: Oral Exam showing hyperplasia of the gingiva. B: Computed tomography (CT) of the neck showing extensive cervial adenopathy. 


\section{Case Reports in Oncology}

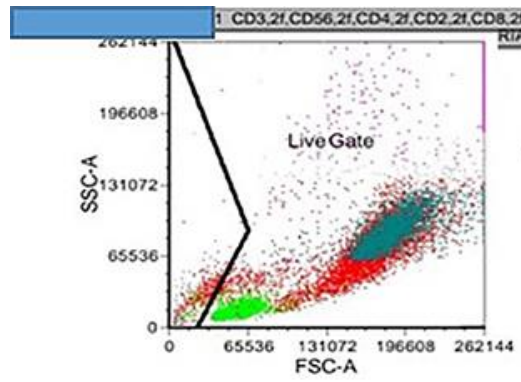

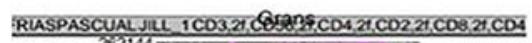
DOI: 10.1159/000494830 (C) 2018 The Author(s). Published by S. Karger AG, Basel www.karger.com/cro

Khaja et al.: Co-Occurrence of Myeloid Sarcoma of the Lymph Node and Acute Monocytic Myeloid Leukemia: A Case Report and Literature Review
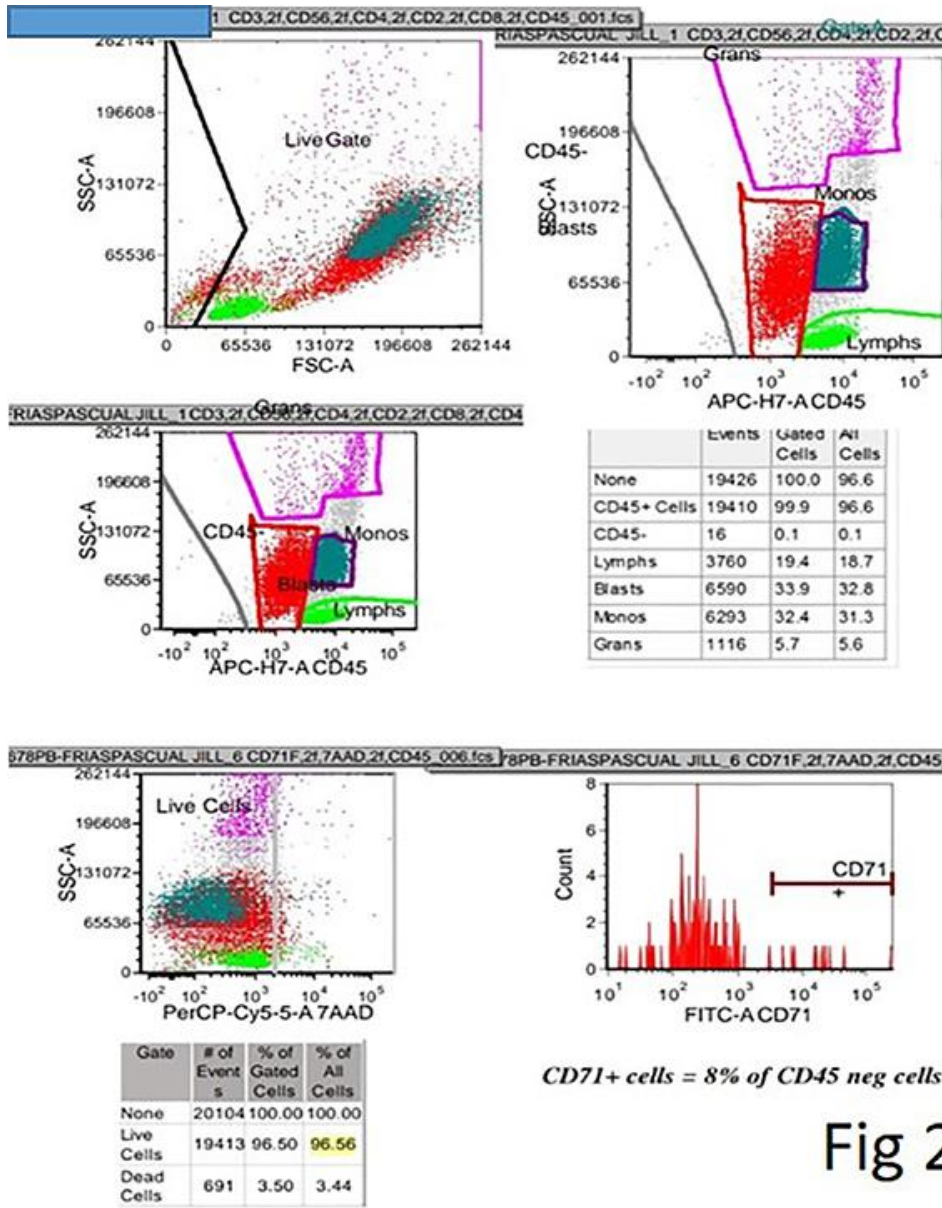

CD7I + cells $=8 \%$ of CD45 neg cells

\section{Fig 2}

Fig. 2. Flow cytometry analysis revealed a CD45 blast-gated population expressing dim CD117, CD4, CD11c, CD13, CD33, CD30, myeloperoxidase (MPO), and HLA-DR, which accounted for approximately $42 \%$ of total gated events. 


\section{Case Reports in Oncology}

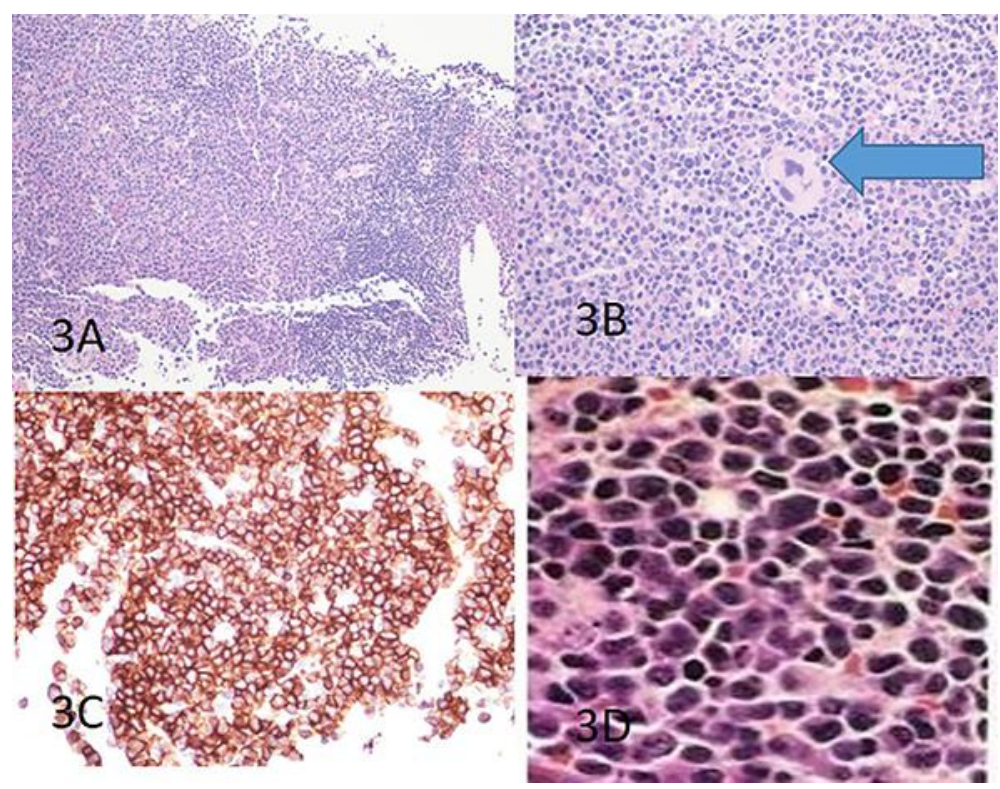

Fig. 3. A: Fine needle aspiration of the cervical lymph node with myeloid sarcoma revealed groups of atypical immature myeloid cells, mixed with occasional megakaryocytes, and eosinophilic myeloid cells infiltrating the lymph node $(\mathrm{H} \& \mathrm{E}$ magnification $\times 200)$. B:On high magnification showing sheets of immature myeloid cells/blasts with admixture of some monoblasts. There is megakaryocyte in the center of Fig( arrow) ( $\mathrm{H}$ and $\mathrm{E}$ magnification $\times 400$ ). C: The neoplastic cells are strongly immunoreactive to CD43 immunohistochemical stain (magnification $\times 400$ ). D: Bone marrow biopsy at the right posterior iliac crest; trephine biopsy showed marked hypercellular marrow, with $>95 \%$ of immature myeloid/monocytic cells, unremarkable bony trabeculae, and no granuloma.

Table 1. Serial measurements of patient complete blood counts

\begin{tabular}{lrrrrrrrrr}
\hline & Day 1 & Day 2 & Day 4 & Day 5 & Day 7 & Day 9 & Day 10 & Day 11 & Day 12 \\
\hline WBC count, K/ $\mu \mathrm{L}$ & 53.5 & 107.0 & 120.0 & 78.9 & 74.0 & 48.0 & 35.0 & 22.0 & 18.0 \\
Hemoglobin, g/dL & 10.8 & 9.4 & 9.2 & 9.0 & 9.6 & 9.7 & 9.0 & 10.1 & 10.4 \\
Hematocrit, \% & 30.9 & 28.0 & 27.7 & 27.0 & 29.0 & 29.1 & 29.2 & 30.0 & 30.0 \\
Platelets, K/ $\mu \mathrm{L}$ & 104.0 & 67.0 & 69.0 & 70.0 & 74.0 & 75.0 & 82.0 & 94.0 & 100.0 \\
\hline
\end{tabular}

